\section{From the authors:}

We would like to thank J. Domagala-Kulawik and colleagues for their interesting and useful comments on our previously published article on T-regulatory (Treg) cells in chronic obstructive pulmonary disease (COPD) [1].

We took great interest in the findings of the study by FIJALKOWSKI et al. [2], which showed that the proportion of Treg cells (identified by flow cytometry with CD4/CD25/ CTLA4 antibodies) was significantly decreased in COPD patients. These results support our conclusions.

Upregulation of Treg cells in central airways, as described in our article, corresponds to the the data of FIJALKOWSKI et al. [2] of an increased Treg cell proportion in patients with inflammatory and tumourous lung disease. Inflammation in central airways is present in practically all smokers. Upregulation of Treg cells could be an adaptive reaction of the immune system to increase the peripheral tolerance against self-antigens produced by continuous irritation of airways by tobacco smoke. At the same time, these changes could be the first step towards tumour genesis, as it is now known that Treg cells decrease cellular immune anti-tumour defence [3]. Opposite changes in peripheral airways in COPD patients which we reported in our article, possibly enhance the autoimmune process [1]. Therefore, the events occurring in central and peripheral airways should be regarded as independent local reactions of the immune system.

We agree that lung tissue taken during surgery from patients with lung cancer may lead to confusing results. However, surgical specimens are the only available material for histopathological examination of small airways and are widely accepted in COPD studies [4].

Using flow cytometry, FIJALKOWSKI et al. [2] found that Treg cell proportion was increased in lung cancer patients which contradicted our results. The authors suggested that Treg cell distribution could be affected by tumour stage and location.

In our study, all subjects had nonsmall cell peripherally located carcinoma at T1 stage (tumour size did not exceed $3 \mathrm{~cm}$ in diameter). The distribution of different histological types of tumour was similar among all the examined groups of patients including the control group. The discrepancy between our results and the results of FIJALKOWSKI et al. [2] could be explained by the methods used for analysis. Flow cytometry of bronchoalveolar lavage (BAL) fluid provides the average proportion of cells from the lung or lobe. A histological analysis reveals the immune cell spectrum in particular tissue areas and therefore reflects local immune events. Of course, it would be intriguing to compare the picture in the areas closer to the tumour location and this could be the task for our future studies.

Other previous studies on Treg cells in COPD confirm our results. Recently, it has been shown that Treg cells are increased in the BAL fluid of healthy smokers and COPD patients, which is consistent with our study results [5]. In addition, it has recently been demonstrated that Treg cells were increased within lymphocyte follicles in moderate COPD [6]. Furthermore, using BAL flow cytometry, BARCELO et al. [7] showed that smokers with preserved lung function had a prominent upregulation of Treg cells that was absent in patients with COPD. In lungs of patients with emphysema the number of $\mathrm{CD} 4{ }^{+} \mathrm{CD} 25^{+}$positive Treg cells was decreased correlating with FOXP3 mRNA expression [8].

In our study [1], lung function testing was performed according to European Respiratory Society and British Thoracic Society guidelines for spirometry [9], and COPD staging according to Global Initiative for Chronic Obstructive Lung Disease criteria [10]. In addition, COPD diagnosis was confirmed by histological examination of lung specimens stained with haematoxylin and eosin to determine structural changes in lung tissue. All COPD patients exhibited characteristic lung tissue changes. For example, in small airways goblet cell metaplasia and mucous plugging, as well as fibrosis and severe inflammation, and in lung parenchyma emphysema and fibrosis was observed. We have not discussed these features in detail in our article as they are widely accepted and used [11]. Therefore, we are sure that airflow limitation was caused by COPD, but not by a solitary peripheral carcinoma.

We agree that the number of nonsmokers enrolled in our study was relatively large. We recruited these patients for a long period of time ( $3 \mathrm{yrs})$. All these subjects really were nonsmokers, not ex-smokers.

To conclude, we would like to thank J. Domagala-Kulawik and colleagues once more for their interesting point of view, comments, suggestions and remarks, which have allowed us to look at our study design from another point of view and provide us with new ideas.

\section{S. Isajevs*, I. Taivans*, G. Strazda*, U. Kopeika", M. Bukovskis ${ }^{\uparrow}$, V. Gordjusina* and A. Kratovska*} *Dept of Pathology, Faculty of Medicine, University of Latvia, \#Centre of Thoracic Surgery, Pauls Stradins Clinical University Hospital, and "Dept of Pulmonology and Allergology, Pauls Stradins Clinical University Hospital, Riga, Latvia.

Correspondence: S. Isajevs, Faculty of Medicine, University of Latvia, 1a Sarlotes street, LV-1001, Riga, Latvia. E-mail: sergisajevs@inbox.lv

Statement of Interest: None declared.

\section{REFERENCES}

1 Isajevs S, Taivans I, Strazda G, et al. Decreased FOXP3 expression in small airways of smokers with COPD. Eur Respir J 2009; 33: 61-67.

2 Fijalkowski R, Domagala-Kulawik J, Dabrowska M, et al. Regulatory cells in COPD patients. Preliminary report. ERS Annual Congress 2008; E4244. www.ers-education.org/lr/ abstract.aspx?idMedia $=77930$.

3 Petersen RP, Campa MJ, Sperlazza J, et al. Tumor infiltrating Foxp3+ regulatory $\mathrm{T}$-cells are associated with recurrence in pathologic stage I NSCLC patients. Cancer 2006; 107: 2866-2872.

4 Saetta M, Baraldo S, Corbino L, et al. CD8+ve cells in the lungs of smokers with chronic obstructive pulmonary disease. Am J Respir Crit Care Med 1999; 160: 711-771. 
5 Smyth LJC, Starkey C, Vestbo J, et al. CD4-regulatory cells in COPD patients. Chest 2007; 132: 156-163.

6 Plumb J, Smyth LJC, Adams HR, et al. Increased T-regulatory cells within lymphocyte follicles in moderate COPD. Eur Respir J 2009; 34: 89-94.

7 Barcelo B, Pons J, Ferrer JM, et al. Phenotypic characterisation of T-lymphocytes in COPD: abnormal CD4+CD25+ regulatory T-lymphocyte response to tobacco smoking. Eur Respir J 2008; 31: $585-562$.

8 Lee SH, Goswami S, Grudo A, et al. Antielastin autoimmunity in tobacco smoking-induced emphysema. Nat Med 2007; 13: 567-569.
9 BTS guidelines for the management of chronic obstructive pulmonary disease. The COPD Guidelines Group of the Standards of Care Committee of the BTS. Thorax 1997; 52: Suppl. 5, S1-S28.

10 Pauwels RA, Buist AS, Calverey MA, et al. Global strategy for the diagnosis, management, and prevention of chronic obstructive pulmonary disease. Am J Respir Crit Care Med 2001; 163: 1256-1276.

11 Saetta M, Turato G, Maestrelli $P$, et al. Cellular and structural bases of chronic obstructive pulmonary disease. Am J Respir Crit Care Med 2002; 163: 1304-1309.

\section{On the issue of exercise normalcy}

\section{To the Editors:}

Cardiopulmonary exercise testing (CPET) has evolved as a useful tool for evaluation of exercise capacity in apparently healthy subjects. In the clinical arena, CPET is widely used to judge exercise "normalcy" in individuals with a suspected disorder and patients with several potential causes of exercise limitation [1]. In order to establish "abnormality", however, it is crucial to obtain representative frames of reference to interpret the (lack of) appropriateness of the systemic responses to exertion. Unfortunately, this is not a trivial task in the nonathletic subject as there are multiple confounding factors, especially behavioural characteristics such as the level of regular physical activity. Not surprisingly, there are only a few sets of reference values for clinical CPET interpretation which have survived the proof of time and are currently used worldwide [1].

In this context, the study recently published in the European Respiratory Journal by $\mathrm{KoCH}$ et al. [2] is welcomed. After evaluating a large sample of apparently healthy males and females with a wide range of age and body dimensions, $\mathrm{KOCH}$ et al. [2] provided a comprehensive set of prediction equations for the main CPET variables. The authors carefully avoided some well-known confounding factors, especially those related to past or current medical conditions, and the statistical analysis was unusually sophisticated.

There is, however, one major shortcoming in the study by $\mathrm{KoCH}$ et al. [2] which might hamper its application in clinical practice. Unfortunately, the participants were not randomly selected from the general population, i.e. they freely volunteered to the study as part of a larger investigation on healthrelated outcomes in Germany. Consequently, it is conceivable that the more active subjects participated, an effect that is likely to be more relevant for the older groups. In fact, the authors stated that "the influence of physical activity was not consistently significant throughout the investigated groups" [2], which suggests that the elderly group were as active as the younger subjects. The hypothesis that the study has been biased to evaluate subjects who were more active than the sedentary, general population is consistent with the higher prevalence of nonsmoking and nonhypertensive subjects in the group of volunteers compared with the complete population $(\mathrm{p}<0.05)$. Moreover, the age-related decline in peak oxygen uptake $\left(V^{\prime} \mathrm{O}_{2}\right)$ was lower than previously reported by most of the previous studies and the predicted values for subjects aged $>40$ yrs were systematically higher than estimated by other commonly used equations. Therefore, age-related decline in predicted $V^{\prime} \mathrm{O}_{2}$ from age 20-25 to 65 yrs has been previously estimated to average $20-25 \%$ in sedentary subjects; in contrast, $\mathrm{KOCH}$ et al. [2] reported only a $15 \%$ decrease in males and females. As a consequence, figure 5 from the study by $\mathrm{KoCH}$ et al. [2] shows that the median peak $V^{\prime} \mathrm{O}_{2}$ values predicted by three other equations for subjects aged $\geqslant 65$ yrs were in the lower quartiles or close to the 5 th percentile in males and females, respectively. Collectively, these findings seem to indicate that the reference values of $\mathrm{KocH}$ et al. [2] are of limited value for the evaluation of exercise normalcy in the specific sub-population of sedentary elderly subjects in whom cardiopulmonary diseases are more prevalent [3] and CPET could be clinically more useful.

We have previously reported the findings of a similar, albeit smaller, study in which the subjects were randomly selected from a database of $>8,000$ subjects [4]. Although this study feature increased enormously the complexity of the investigation, it eventually proved essential to obtain truly representative data for clinical interpretation of CPET. For instance, occasional volunteers were submitted to the same evaluation protocol but results were not considered on the final analysis. As expected, they were more active, fitter and leaner than the randomised subjects. In fact, if data from the nonrandomised subjects had been included in the analysis, predicted peak $V^{\prime} \mathrm{O}_{2}$ values would be almost $20 \%$ higher, i.e. values quite similar to those reported by Koch et al. [2]. In our view, this is the main reason that explains why our prediction equations provide the lowest peak $V^{\prime} \mathrm{O}_{2}$ values amongst other sources of reference values, e.g. fig. 5 from $\mathrm{KocH}$ et al. [2]. Moreover, we developed reference values for several effort-independent, submaximal relationships obtained in the incremental phase of exercise [5]. Considering that these variables are less influenced by maximal aerobic capacity, $\mathrm{KoCH}$ et al. [2] might have excellent material in their hands to further contribute to the field.

In conclusion, it is our opinion that the "ideal" set of reference values for clinical interpretation of CPET is still to be generated. Although such investigation would certainly share 